\title{
La investigación científica y la educación superior: factores relevantes de los sistemas de innovación
}

\author{
Edna Karina Alcázar Farías*, Alejandro Lozano Guzmán**
}

Resumen: En este trabajo se presenta una revisión del concepto de innovación y la descripción de los diferentes modelos de innovación propuestos por diversos autores. De esta revisión, se seleccionan los modelos de caja negra, de relación en cadena y las recomendaciones plasmadas en la $3^{\mathrm{a}}$ edición del Manual de Oslo de la OCDE para desarrollar un modelo numérico que permite simular el impacto que la inversión en ciencia y tecnología tiene sobre la sociedad. El modelo desarrollado se aplica para una región que se denomina el Sistema de Innovación Regional de Querétaro (SIRQ), tomando en cuenta el contexto de la situación de la ciencia y la tecnología en México. Para este sistema, se señalan los diferentes factores que se consideran como entradas y salidas del modelo y se explican los valores asignados a cada uno de estos factores. Buscando ilustrar de forma objetiva el potencial de uso que tiene el modelo propuesto, se analizan seis escenarios para la región seleccionada como ejemplo.

Palabras clave: Ciencia, tecnología, modelos de innovación, impacto.

\section{Scientific research and higher education as relevant factors in innovation systems}

Abstract: This paper presents the concept of innovation and the description of various innovation models put forward by different authors. Three models were selected —black box, chain relations and the recommendations from the $3^{\text {rd }}$ edition of the OECD's Oslo Manual - in order to develop a numerical model for simulating the impact on society of investments in science and technology. Taking into account Mexico's SET context, the developed model was applied to the region called Queretaro Regional Innovation System (SIRQ). For this system, input-output factors are defined, with numerical values assigned to them based on local conditions. The model is applied by simulating six scenarios for possible development of SIRQ, considering the influence of scientific research and higher education on the region's development.

Keywords: Science, technology, innovation models, impact.

* Universidad Autónoma de Querétaro, Facultad de Ingeniería, México. Correo-e: edna.alcazar@ uaq.mx.

** Centro de Ciencia Aplicada y Tecnología Avanzada, Unidad Querétaro, Instituto Politécnico Nacional, México. Correo-e:alozano@ipn.mx.

Recibido: 14-09-2011; 2. ${ }^{a}$ versión: 27-01-2012; aceptado: 10-02-2012. 


\section{Introducción}

La innovación, de acuerdo a la $3^{\text {a }}$ edición del manual de Oslo de la OCDE (OCDE, 2005), se define como la puesta en práctica de un producto (bien o servicio) nuevo o significativamente mejorado, de un proceso, de un nuevo método de comercialización o de un nuevo método organizacional del sitio de trabajo o en las relaciones externas. De forma amplia se puede decir que el concepto de innovación engloba la creación y/o mejora de los productos y/o procesos que realizan las instituciones (de cualquier tipo), y puede estar presente en cualquier ámbito de la organización (Edquist, 2001). Asimismo, cabe destacar que el proceso de innovación no es lineal, sino que se trata de un proceso interactivo e iterativo (Lundvall, 1985; Nelson, 1993; Edquist, 1997; Edquist y Mckelvey, 2000). Por otra parte se reconoce que la producción de conocimiento y las actividades innovadoras se tienden a aglomerar geográficamente (Breschi y Malerba, 1997; Cooke y otros, 1997; Freeman, 1995). En el análisis de los sistemas de innovación (SI), se utilizan aproximaciones diferentes dependiendo de la unidad de estudio que se desea utilizar. A nivel de un país completo se tendría la noción de Sistemas Nacionales de Innovación (Freeman, 1988), a nivel de un sector productivo en específico se considerarían los Sistemas Sectoriales de Innovación (Carlsson, 1995), o a nivel de territorios más acotados y específicos se manejaría la noción de Sistemas Locales o Regionales de Innovación (Braczyk y otros, 1998).

Entre las teorías y modelos de innovación, se encuentran el modelo de caja negra, que trata de explicar la adquisición de conocimiento y tecnología a partir de las interacciones de la empresa con los clientes y proveedores. En este modelo, la transferencia de conocimiento, abarca desde la propiedad intelectual hasta la transferencia de conocimiento entre equipos de trabajo (Desai, 2006). Este modelo vislumbra a la innovación como un proceso de insumo-producto, tal y como se muestra en la figura 1.

\section{FIGURA 1}

Modelo lineal del vínculo entre el conocimiento y el desempeño económico

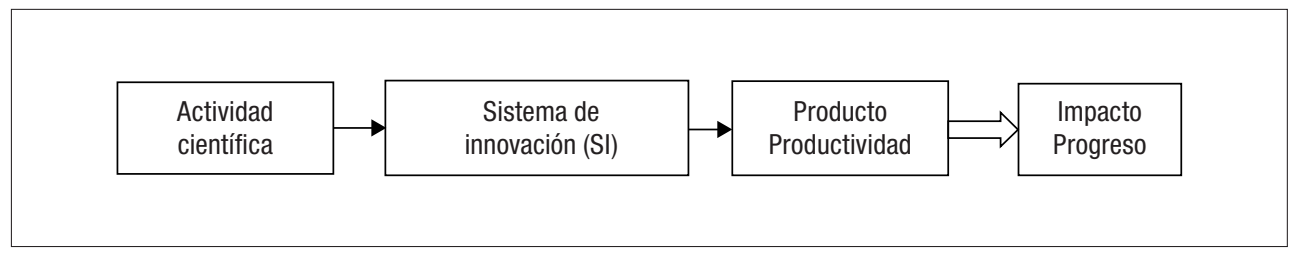

Fuente: elaboración propia.

La otra concepción teórica en la que se enmarcan los modelos de innovación es el modelo de relación en cadena, el cual se describe como un modelo 
interactivo del proceso que va del conocimiento a la aplicabilidad. Entre estos modelos se incluye el de triple hélice que considera a la educación superior y a la investigación científica agrupados en la universidad, a la industria y al gobierno como hélices del proceso de innovación (Etzkowitz y Leydesdorff, 2000). Más recientemente ya se habla del modelo de cuatro hélices (Alfonso y otros, 2010), el cual, adicionalmente a lo anterior, incluye a la sociedad como agente de los sistemas nacionales y regionales de innovación (Lundvall y Nielsen, 2007) y la generación de empresas de base tecnológica como resultado de los llamados "Spin-Off» universitarios (Rohrbeck y otros, 2009). En estos modelos el proceso de innovación se caracteriza por interacciones y efectos de ida y vuelta, así como por las numerosas interacciones que ligan la ciencia, la tecnología y la innovación, como se muestra en la figura 2. En este mismo sentido de relaciones bidireccionales, se dan las interacciones entre los componentes del SI propuesto en la $3^{\text {a }}$ edición del Manual de Oslo (figura 3). Considerando los modelos de caja negra, de relación en cadena y las interacciones definidas en la $3^{\text {a }}$ edición del Manual de Oslo, se desarrolló el modelo que permite analizar la influencia de la educación superior y la investigación científica en el proceso de innovación y su impacto en la sociedad, que se presenta en este trabajo.

FIGURA 2

Modelo de relación en cadena

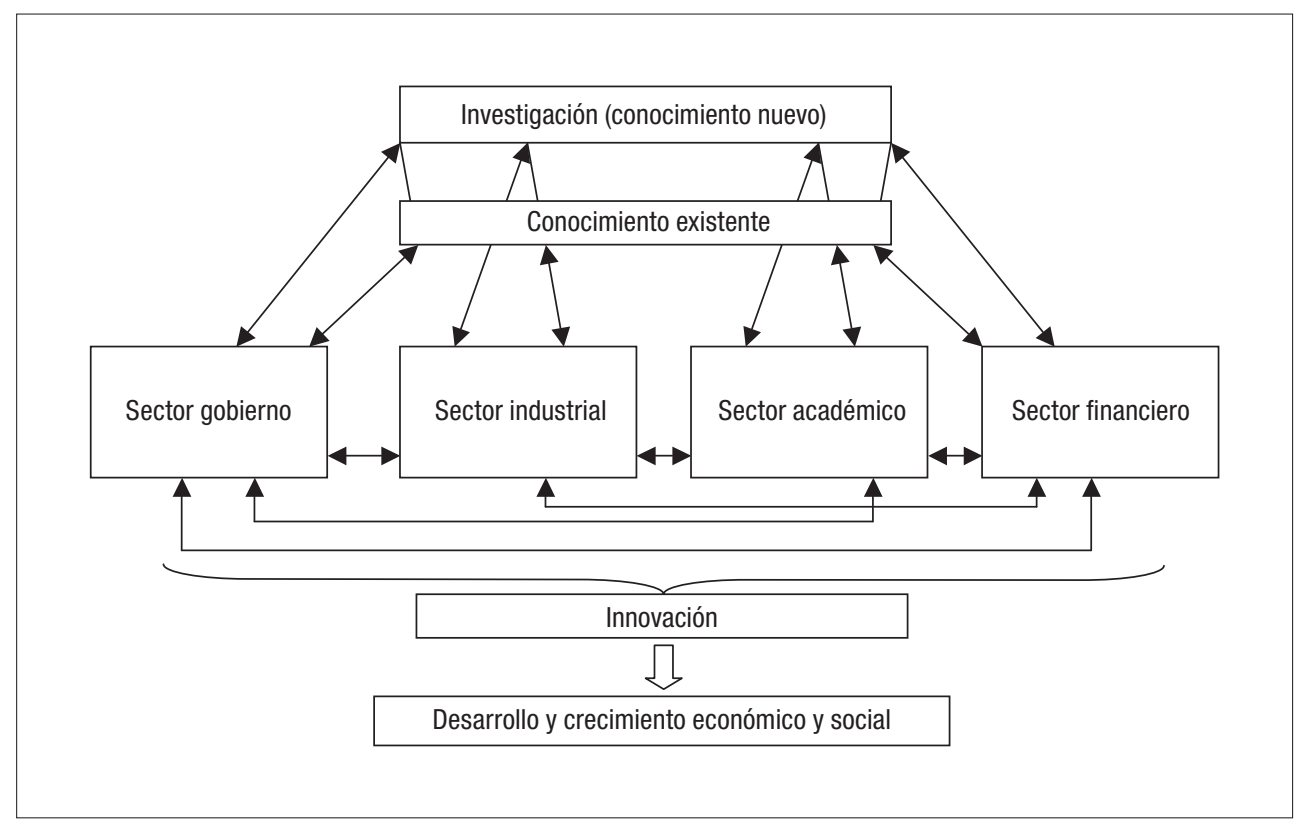

Fuente: adaptación del modelo de relación en cadena de OCDE. 
FIGURA 3

Interacciones entre componentes de un SI

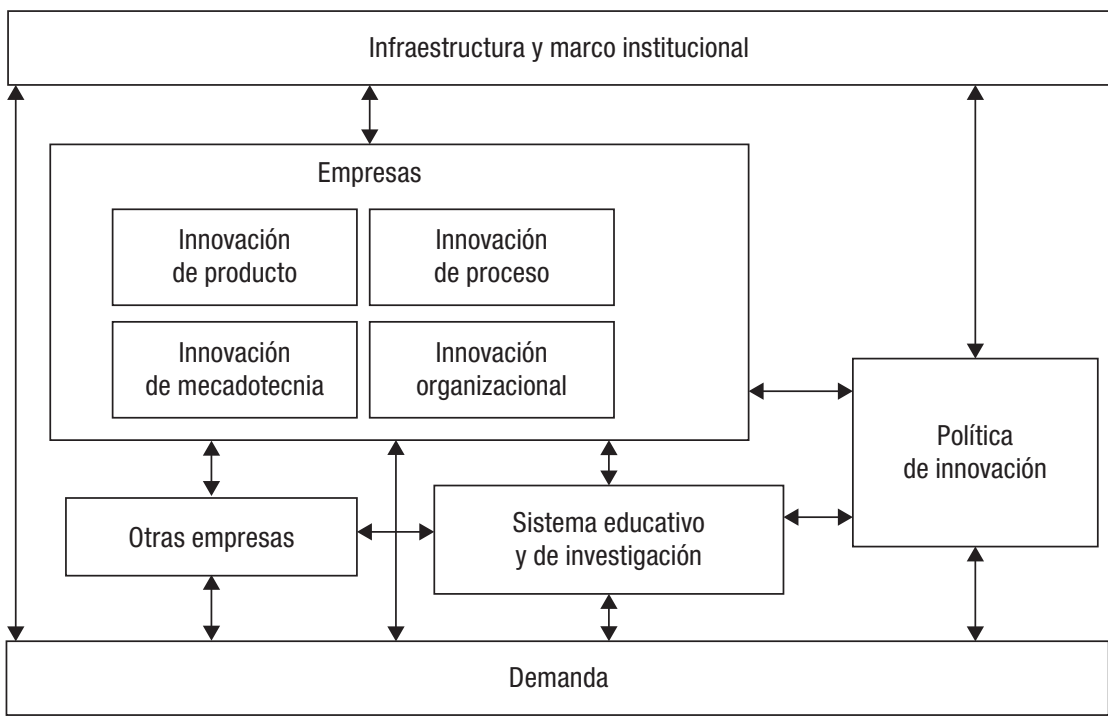

Fuente: organización para la Cooperación y el Desarrollo Económico (OECD), 2005.

\section{Panorama de la situación de la ciencia y tecnología en México}

Como se puede apreciar en la figura 3, se considera que la educación y la investigación son parte fundamental de un sistema de innovación. No obstante, es claro que si las relaciones entre la investigación con las demandas de la sociedad, las empresas y las políticas de innovación no están correctamente articuladas, el sistema no funciona. Tal es el caso de lo que sucede en México.

Considerando que tanto para enseñar como para investigar se invierten recursos humanos, recursos monetarios, recursos materiales y otros, es fundamental conocer la forma en que estos recursos son invertidos, así como los beneficios académicos, económicos y sociales que se obtienen. Con base en la premisa de que a mayor inversión en educación, ciencia y tecnología, mayor desarrollo, como se ha comprobado en varios países, aquí se presentan una serie de escenarios del posible impacto que tiene la investigación para el desarrollo de México. Con estos escenarios, se busca dar pistas de por qué después de más de 25 años de supuesta política de apoyo al desarrollo científico y tecnológico, la ciencia y la 
tecnología siguen siendo en México un «lujo académico» (Lozano, 2003, 2008). Esto es palpable al observar que el estado de la ciencia y la tecnología en este país es prácticamente el mismo que hace 25 años (Lozano, 2009). Esto es, el sector académico se encuentra inmerso en su propio espacio, el sector industrial es dependiente de tecnología adquirida en el extranjero, el sector gubernamental se mantiene ajeno y no ve a la ciencia y la tecnología como un motor para el desarrollo socioeconómico y, finalmente, el sector financiero no ve a los proyectos de ciencia y tecnología como una fuente de inversión productiva. La diferencia entre la situación de hace 25 años y la actual estriba en que en el pasado había pocos centros de investigación y pocos investigadores, mientras que en la actualidad, bajo las mismas condiciones ya descritas, se cuenta con una mayor cantidad de docentes y de investigadores, y más empresas, pero, en general, siguen estando aislados sin expectativas reales de vinculación y generación de riqueza (Muñoz y otros, 2001).

\section{Descripción del modelo}

Considerando los modelos propuestos por los diversos autores referenciados principalmente el de caja negra, el de relación en cadena y las recomendaciones plasmadas en la $3^{\text {a }}$ edición del Manual de Oslo de la OCDE, se puede decir que el proceso de innovación es sistemático, por tanto puede ser entendido, aprendido y replicado. Por esto, el proceso en sí mismo puede ser representado, a su vez, por un modelo que describa las interacciones y la dinámica del sistema (Sancho, 2007). En la figura 4 se muestran las interacciones entre la academia, el sector económico, la sociedad y el gobierno, consideradas en el modelo desarrollado.

En este modelo se relacionan la educación superior (IES) y la investigación (CI), con diversos sectores que inciden en el desarrollo socioeconómico de México. A partir de estas relaciones, se estudia la influencia que estos factores tienen en el proceso de transferencia de conocimiento científico y tecnológico a los usuarios y su impacto en el desarrollo industrial y social del país (IS).

Se planteó un modelo en el que se considera que la ciencia y el desarrollo tecnológico generan desarrollo socioeconómico, en la medida que la investigación produce bienestar social a través del sistema de innovación (SI) y no solo productos de carácter académico. El modelo requiere contar con información sobre el flujo del conocimiento del SI, así como de indicadores científicos, tecnológicos, sociales y económicos, y del mapeo del sistema de educación superior, los centros de investigación y los sectores económicos.

Las interrelaciones entre los diversos actores involucrados en el proceso a seguir para que la investigación se convierta en un beneficio tangible para la sociedad en su conjunto, se plantearon empleando el paquete de simulación VENSIM (Ventana ${ }^{\circledR}$ Systems Incorporated, Harvard, MA, Estado Unidos). La tabla I lista los factores considerados en el modelo. 


\section{FIGURA 4}

\section{Modelo: El Sistema de Innovación para una determinada región}

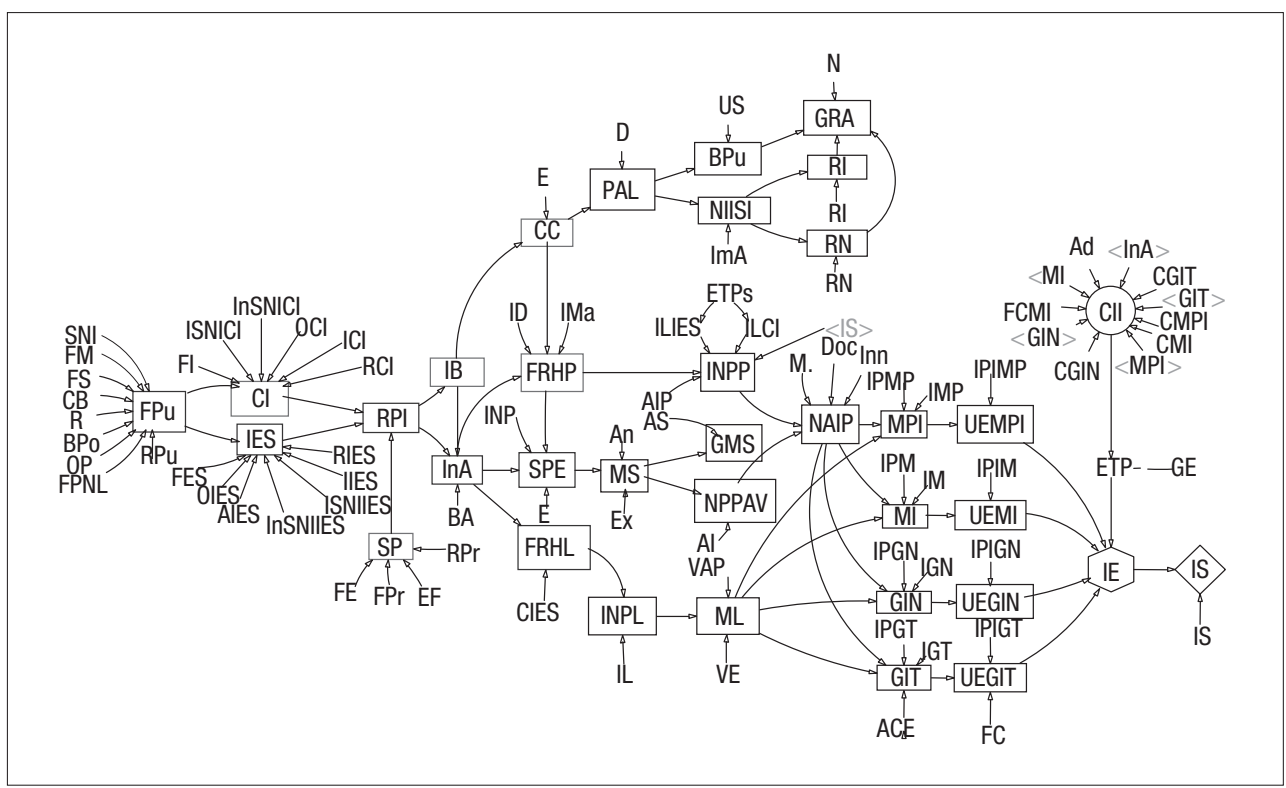

Fuente: elaboración propia.

TABLA I

Factores considerados en el modelo

\begin{tabular}{|c|c|c|c|}
\hline Variable & Código & Variable & Código \\
\hline ACE & Atracción de capitales extranjeros & ILIES & Inserción laboral en IES \\
\hline $\mathrm{Ad}$ & Aplicabilidad & IM & Impacto mediana \\
\hline $\mathrm{AI}$ & Aplicación industrial & $\operatorname{Im} A$ & Impacto académico \\
\hline AIES & Alumnos IES & IMa & Impacto maestría \\
\hline AIP & Aceptación industrial del posgrado & IMP & Impacto micro y pequeña \\
\hline An & Aplicación & InA & Investigación aplicada \\
\hline AS & Aplicación social & Inn & Innovación \\
\hline BA & Básica a Aplicada & INP & Inserción nivel posgrado \\
\hline BPo & Becas posgrado & INPL & $\begin{array}{l}\text { Impacto en el nivel profesional de } \\
\text { los estudiantes licenciatura }\end{array}$ \\
\hline $\mathrm{BPu}$ & Beneficiarios de las publicaciones & INPP & $\begin{array}{l}\text { Impacto en el nivel profesional de } \\
\text { los estudiantes posgrado }\end{array}$ \\
\hline $\mathrm{C}$ & Capacitación & InSNICI & Investigadores no SNI CI \\
\hline $\mathrm{CB}$ & Ciencia básica & InSNIIES & Investigadores no SNI IES \\
\hline
\end{tabular}


TABLA I (continuación)

\begin{tabular}{|c|c|c|c|}
\hline Variable & Código & Variable & Código \\
\hline $\mathrm{CC}$ & Conocimiento científico & IPGN & Impacto posgrado gran nacional \\
\hline CGIN & $\begin{array}{l}\text { Conectividad con la gran industria } \\
\text { nacional }\end{array}$ & IPGT & Impacto posgrado gran trasnacional \\
\hline CGIT & $\begin{array}{l}\text { Conectividad con la gran industria } \\
\text { trasnacional }\end{array}$ & IPIGN & $\begin{array}{l}\text { Incremento de productividad por in- } \\
\text { vestigación gran nacional }\end{array}$ \\
\hline $\mathrm{CI}$ & Centros de investigación & IPIGT & $\begin{array}{l}\text { Incremento de productividad por in- } \\
\text { vestigación gran trasnacional }\end{array}$ \\
\hline CIES & Capacitación en IES & IPIM & $\begin{array}{l}\text { Incremento de productividad por in- } \\
\text { vestigación mediana }\end{array}$ \\
\hline CIl & Conectividad intersecretarial & IPIMP & $\begin{array}{l}\text { Incremento de productividad por in- } \\
\text { vestigación micro y pequeña }\end{array}$ \\
\hline CMI & $\begin{array}{l}\text { Conectividad con la mediana indus- } \\
\text { tria }\end{array}$ & IPM & Impacto posgrado mediana \\
\hline CMPI & $\begin{array}{l}\text { Conectividad con la micro y peque- } \\
\text { naa industria }\end{array}$ & IPMP & Impacto posgrado micro y pequeña \\
\hline $\mathrm{D}$ & Divulgación & IS & Impacto social \\
\hline Doc & Doctorado & ISNICI & Investigadores SNI CI \\
\hline E & Eficiencia & ISNIIES & Investigadores SNI IES \\
\hline $\mathrm{EF}$ & Estímulos fiscales & M & Maestría \\
\hline ETPs & Eficiencia terminal posgrado & MI & Mediana industria \\
\hline ETP & Empresas con tecnología propia & ML & Mercado laboral \\
\hline Ex & Éxito & MPI & Micro y pequeña industria \\
\hline FC & Fuga de capital & MS & Mejora sectorial \\
\hline $\mathrm{FE}$ & Fondos del exterior & $\mathrm{N}$ & Negocios \\
\hline FES & Financiamiento educación superior & NAIP & $\begin{array}{l}\text { Nivel de aceptación industrial del } \\
\text { posgrado }\end{array}$ \\
\hline FI & Fondos para infraestructura & NIISI & Nivel de impacto ISI \\
\hline FM & Fondos mixtos & NPPAV & $\begin{array}{l}\text { Nuevos productos y procesos de alto } \\
\text { valor agregado }\end{array}$ \\
\hline FPNL & Financiamiento privado no lucrativo & OCI & Otros CI \\
\hline FPr & Financiamiento privado & OIES & Otros IES \\
\hline $\mathrm{FPu}$ & Financiamiento público & OP & Otros programas \\
\hline FRHL & $\begin{array}{l}\text { Formación de recursos humanos li- } \\
\text { cenciatura }\end{array}$ & PAL & Publicación de artículos y libros \\
\hline FRHP & $\begin{array}{l}\text { Formación de recursos humanos ni- } \\
\text { vel posgrado }\end{array}$ & $\mathrm{R}$ & Repatriación \\
\hline FS & Fondos sectoriales & RCI & Reinversión en CI \\
\hline GE & Generación de empresas & RI & Reconocimiento internacional \\
\hline
\end{tabular}


TABLA I (continuación)

\begin{tabular}{l|l|l|l}
\hline Variable & \multicolumn{1}{|c|}{ Código } & Variable & \multicolumn{1}{c}{ Código } \\
\hline GIN & Gran industria nacional & RIES & Reinversión en IES \\
\hline GIT & Gran industria transnacional & RN & Reconocimiento nacional \\
\hline GMS & Gobierno mejores servicios & RPI & $\begin{array}{l}\text { Recursos para proyectos de investi- } \\
\text { gación }\end{array}$ \\
\hline GRA & Generación de recursos académicos & RPr & Reinversión privada \\
\hline IB & Investigación básica & RPu & Reinversión pública \\
\hline ICI & Infraestructura CI & SNI & Sistema Nacional de Investigadores \\
\hline ID & Impacto doctorado & SP & Sector privado \\
\hline IE & Impacto económico & SPE & Solución de problemas específicos \\
\hline IES & Instituciones de Educación Superior & UEGIN & $\begin{array}{l}\text { Utilidad económica gran industria } \\
\text { nacional }\end{array}$ \\
\hline IGN & Impacto gran nacional & UEGIT & $\begin{array}{l}\text { Utilidad económica gran industria } \\
\text { transnacional }\end{array}$ \\
\hline IGT & Impacto gran trasnacional & UEMI & $\begin{array}{l}\text { Utilidad económica mediana indus- } \\
\text { tria }\end{array}$ \\
\hline IIES & Infraestructura IES & UEMPI & $\begin{array}{l}\text { Utilidad económica micro y pequeña } \\
\text { industria }\end{array}$ \\
\hline IL & Inserción laboral & US & Utilidad social \\
\hline ILCI & Inserción laboral en CI & VAP & Valor agregado personal \\
\hline & & VE & Valoración empresarial \\
\hline
\end{tabular}

Fuente: elaboración propia.

El modelo requiere como entrada los siguientes datos, que se publican anualmente: el financiamiento público (FPu) que está compuesto por la inversión que se hace en fondos para desarrollo de infraestructura, el financiamiento para investigación en las IES, el Sistema Nacional de Investigadores (SNI) y los diferentes fondos de financiamiento que promueve el Consejo Nacional de Ciencia y Tecnología (CONACYT) a saber: fondos mixtos (FM), fondos sectoriales (FS), de ciencia básica (CB), de retención y repatriación (R), para becas de posgrado (BPo) y otros programas (OP) que han surgido para atender necesidades específicas de una región. Se eligieron estos factores como entradas, ya que en la última década el financiamiento público de la ciencia y la tecnología en México se ha realizado a través de los programas del CONACYT. Por lo que respecta al financiamiento privado (FPr), se consideró que éste está compuesto por los fondos que llegan de otros países (FE), por los estímulos que el gobierno ofrece al sector privado $(\mathrm{EF})$ y por el dinero que aportan para investigación las propias empresas (RPr). Se hace énfasis en que las aportaciones económicas provenientes del sector privado (SP), están directamente relacionadas con proyectos de investigación 
que tengan pertinencia con la solución de situaciones que interesan, precisamente, al sector privado.

En el modelo se hace diferencia entre los fondos públicos (FPu) que se canalizan a las IES y los que van a los CI. Se realiza dicha diferenciación porque se considera que aunque en ambos tipos de institución se realiza investigación, los objetivos y las relaciones con el entorno industrial son diferentes en cada caso. Por la misma razón, también se hace diferencia entre los fondos públicos que se emplean en investigación básica (IB) y en investigación aplicada (InA).

Los otros factores que se encuentran en esta primera parte del modelo son los factores que cuantifican la eficiencia de la variable que afectan. Dichos valores van de 0 a 1 , donde 0 significa total ineficiencia y 1 eficiencia total. Lo anterior aplica para todos los factores utilizados en el modelo.

A partir de que los recursos son divididos en investigación básica (IB) o aplicada (InA) se consideran dos ramas para los resultados obtenidos: académicos y económicos.

Uno de los factores que tiene una influencia importante en el modelo es el factor de investigación básica a aplicada (BA), el cual cuantifica la medida en que los conocimientos desarrollados en una determinada región son usados para beneficio de la misma, ya sea solucionando problemas o creando productos y procesos innovadores.

El primer resultado que se obtiene de la investigación básica (IB) es el conocimiento científico (CC), el cual por su naturaleza, tiene un valor que no puede cuantificarse por completo, por lo que cabe aclarar que en el modelo no se le da un valor monetario, sino que se considera la eficiencia (E) con la cual este conocimiento es utilizado, ya sea en el mismo aspecto académico o por los diversos sectores industriales de la región.

De la investigación aplicada (InA) se obtiene como resultado principalmente la solución a problemas específicos (SPE) de una región y la implementación de nuevos productos o procesos (NPPAV). Una vez establecido lo anterior, el modelo considera el movimiento y evolución que tiene el dinero invertido en el desarrollo de conocimiento científico (CC). De esta manera, se toma en cuenta que uno de los aspectos más importantes y reconocidos, tanto a nivel internacional como nacional para evaluar el impacto del conocimiento científico, es la publicación de artículos y libros, participación en congresos y las citas en revistas indizadas (PAL). Aunque en este proyecto, se reconoce la importancia de éstas últimas, no se consideraron como factores, pues se estima que benefician a los autores e instituciones de forma más bien individual que a la región como un todo. No obstante, sí se toma en cuenta el reconocimiento tanto nacional (RN) como internacional (RI), pues ambos son factores a considerar para la obtención de recursos financieros para realizar investigación $(\mathrm{N})$.

Por otra parte, se considera la influencia económica que puede tener en la región la difusión de los conocimientos en revistas y libros, pero desde el punto de vista de la utilidad social (BPu). Lo anterior, tomando en cuenta la factibilidad 
que tenga dicho conocimiento para que pueda ser utilizado por alguna empresa para generar riqueza.

El modelo desarrollado en esta investigación considera la especialización de la región en ciertas áreas del conocimiento y ciertas tecnologías, pues se estima que después de varias generaciones, se tiene un mayor crecimiento y desarrollo si hay un cierto grado de especialización. En el factor mejora sectorial (MS), se representa esta especialización a través, ya sea de nuevos productos y procesos de alto valor agregado (NPPAV), y/o en una mejor atención de necesidades sociales como salud, educación, y transporte, entre otros (GMS).

Por una parte, la solución de problemas específicos (SPE), la creación de productos y procesos (NPPAV), y en general, un enfoque de innovación (Inn), generan un mayor interés por parte de los sectores industriales tanto en los programas de posgrado como en sus egresados (NAIP). Lo que trae como consecuencia una mayor inversión en proyectos de investigación.

Como ya se mencionó anteriormente, en el modelo se consideró como los ejes estructurales del sistema a la academia, la empresa y al gobierno y alrededor de éstos a la sociedad. En este sentido, cuando las empresas (MPI, MI, GIN, GIT) se ven impactadas positivamente como resultado de desarrollos científicos y tecnológicos (IPGN, IPMP, IPM, IPGT) y sus utilidades se ven beneficiadas (IPIGN, IPIGT, IPIM, IPIMP) se supone una derrama económica que apoya directamente a la sociedad al crearse más empleos mejor pagados (ML). De esta forma, la región se convierte en un polo de atracción que capta al mejor personal, a mejores empresas, a mejores servicios y a un mayor compromiso por parte del gobierno; quien por su lado, puede verse beneficiado, con proyectos de investigación que realmente ayuden en la solución de los problemas que afectan a la población de la región (GMS).

Finalmente, se estima que después de algunos ciclos, se forman redes que conectan a todos los actores que participan en la vida económica, política y social de la región (CII). Dicha conectividad interinstitucional, trabajando con metas y objetivos comunes, genera desarrollo y crecimiento para los diferentes estratos sociales, generando una mejor distribución de la riqueza.

\section{Resultados y discusión}

Como ejemplo de aplicación del modelo desarrollado en el contexto de México, se recabaron los datos del Sistema de Innovación Regional de Querétaro (SIRQ), pues se reconoce que la región, ubicada en el centro del país, cuenta con la capacidad y las infraestructuras necesarias para impulsar el desarrollo económico y la competitividad con base en actividades científicas y tecnológicas. La selección de la localidad se basa en que Querétaro es una ciudad con un gran dinamismo industrial, con empresas mexicanas (GIN) y transnacionales de clase mundial (GIT), industria mediana (MI) y pequeña (MPI), centros públicos de investigación y desarrollo tecnológico (CI), universidades (IES), instituciones finan- 
cieras, y dependencias gubernamentales. Para esta región se simularon seis escenarios. Los valores de cada factor se obtuvieron de una serie de reuniones con 10 líderes de opinión de la industria, el gobierno, la academia y el sector financiero de la región, a quienes se les cuestionó que calificaran entre 0 y 1 cada uno de los factores listados en los diferentes escenarios analizados (Lozano, 2008). El promedio de estas calificaciones se indica en la tabla II.

\section{Escenario ideal:}

Caso A: este escenario se caracteriza por tener una eficiencia total, es decir, los valores de cada uno de los factores es 1. Asimismo, existe una conectividad interinstitucional bien definida y funcional. Este escenario sería el ideal, porque como se verá más adelante, es el que trae más beneficios a la región.

Caso B: este escenario se caracteriza por tener una eficiencia total, al igual que el escenario anterior, pero en este caso no existe conectividad interinstitucional, lo cual ocasiona que los beneficios aunque muy evidentes y satisfactorios, son mucho menores que en el escenario optimista A.

2. Escenario real: en este escenario se busca reflejar el caso de una realidad óptima, es decir, considerar que el sistema trabaja con factores de eficiencia máxima, tomando en cuenta las limitaciones inherentes a cada una de las instituciones y actores participantes. En este caso, cada actor tiene una mentalidad de cooperación mutua, se trabaja de forma colectiva, dejando a un lado los intereses individuales. Se tienen objetivos y metas específicas que impulsan el crecimiento y desarrollo de la región en conjunto.

3. Escenario realista: considera que los factores toman un valor más realista desde el punto de vista actual del sistema de ciencia y tecnología que se ha estado desarrollando en la región de Querétaro. En este escenario, se observa que mientras algunas instituciones o actores y sus interrelaciones trabajan con cierta eficiencia, otras lo hacen con un grado de ineficiencia.

4. Escenario pesimista: este escenario tiene como característica la falta de objetivos y metas comunes para la región en conjunto. Lo que ocasiona que la interacción entre los diferentes actores sea, en algunos casos, prácticamente nula. Por tanto, se tiene ineficiencia tanto al interior como al exterior de cada actor, por la falta de coordinación y de una visión común de innovación. Este escenario es individualista y se buscan soluciones de corto plazo. Lo que perjudica el crecimiento de la región.

5. Escenario de ineficiencia: este es el peor caso, pero se aclara que se trata de un escenario supuesto, pues considera que se trabaja bajo total ineficiencia, es decir, cada factor toma un valor de cero, lo que ocasiona que se pierda toda la inversión y no se tenga beneficio alguno. 
TABLA II

Valores de los factores para cada escenario considerado

\begin{tabular}{|c|c|c|c|c|c|c|}
\hline \multicolumn{2}{|r|}{ Escenario } & \multirow{2}{*}{\begin{tabular}{|c|} 
Ideal \\
1 \\
\end{tabular}} & \multirow{2}{*}{$\begin{array}{c}\text { Real } \\
0,8 \\
\end{array}$} & \multirow{2}{*}{$\begin{array}{c}\text { Realista } \\
0,4\end{array}$} & \multirow{2}{*}{$\begin{array}{c}\text { Pesimista } \\
0,1\end{array}$} & \multirow{2}{*}{$\begin{array}{c}\text { Ineficiencia } \\
0\end{array}$} \\
\hline 1 & Aceptación industrial del posgrado & & & & & \\
\hline 2 & Alumnos de IES & 1 & 0,9 & 0,7 & 0,4 & 0 \\
\hline 3 & Aplicabilidad & 1 & 0,9 & 0,3 & 0,1 & 0 \\
\hline 4 & Aplicación & 1 & 0,8 & 0,3 & 0,05 & 0 \\
\hline 5 & Aplicación industrial & 1 & 0,7 & 0,2 & 0,05 & 0 \\
\hline 6 & Aplicación social & 1 & 0,8 & 0,2 & 0,1 & 0 \\
\hline 7 & Atracción de capitales extranjeros & 1 & 0,8 & 0,4 & 0,1 & 0 \\
\hline 8 & Básica a Aplicada & 1 & 0,6 & 0,4 & 0,1 & 0 \\
\hline 9 & Capacitación & 1 & 0,8 & 0,1 & 0,05 & 0 \\
\hline 10 & Capacitación en IES & 1 & 0,7 & 0,1 & 0,04 & 0 \\
\hline 11 & $\begin{array}{l}\text { Conectividad con la gran industria na- } \\
\text { cional }\end{array}$ & 1 & 0,9 & 0,5 & 0,2 & 0 \\
\hline 12 & $\begin{array}{l}\text { Conectividad con la gran industria } \\
\text { trasnacional }\end{array}$ & 1 & 0,9 & 0,6 & 0,2 & 0 \\
\hline 13 & Conectividad con la mediana industria & 1 & 0,8 & 0,4 & 0,2 & 0 \\
\hline 14 & $\begin{array}{l}\text { Conectividad con la micro y pequeña } \\
\text { industria }\end{array}$ & 1 & 0,7 & 0,3 & 0,2 & 0 \\
\hline 15 & Divulgación & 1 & 0,9 & 0,5 & 0,2 & 0 \\
\hline 16 & Doctorado & 1 & 0,7 & 0,2 & 0,05 & 0 \\
\hline 17 & Eficiencia & 1 & 0,5 & 0,2 & 0,05 & 0 \\
\hline 18 & Eficiencia terminal posgrado & 1 & 0,8 & 0,4 & 0,1 & 0 \\
\hline 19 & Estudiantes de licenciatura IES & 1 & 0,8 & 0,7 & 0,5 & 0 \\
\hline 20 & Éxito & 1 & 0,8 & 0,3 & 0,1 & 0 \\
\hline 21 & Fuga de capitales & 1 & 0,1 & 0,5 & 0,9 & 0 \\
\hline 22 & Generación de empresas & 1 & 0,9 & 0,4 & 0,1 & 0 \\
\hline 23 & Impacto académico & 1 & 0,9 & 0,6 & 0,1 & 0 \\
\hline 24 & Impacto doctorado & 1 & 0,9 & 0,7 & 0,4 & 0 \\
\hline 25 & Impacto gran nacional & 1 & 0,8 & 0,4 & 0,2 & 0 \\
\hline 26 & Impacto gran trasnacional & 1 & 0,7 & 0,3 & 0,05 & 0 \\
\hline 27 & Impacto maestría & 1 & 0,9 & 0,6 & 0,2 & 0 \\
\hline 28 & Impacto mediana & 1 & 0,8 & 0,6 & 0,2 & 0 \\
\hline 29 & Impacto micro y pequeña & 1 & 0,9 & 0,7 & 0,3 & 0 \\
\hline 30 & Impacto posgrado gran nacional & 1 & 0,5 & 0,2 & 0,05 & 0 \\
\hline 31 & Impacto posgrado gran trasnacional & 1 & 0,6 & 0,3 & 0,1 & 0 \\
\hline 32 & Impacto posgrado mediana & 1 & 0,7 & 0,3 & 0,1 & 0 \\
\hline 33 & Impacto posgrado micro y pequeña & 1 & 0,5 & 0,2 & 0,05 & 0 \\
\hline
\end{tabular}


TABLA II (continuación)

\begin{tabular}{|c|c|c|c|c|c|c|}
\hline \multicolumn{2}{|c|}{ Factor } & \multirow{2}{*}{\begin{tabular}{|c|} 
Ideal \\
1 \\
\end{tabular}} & \multirow{2}{*}{$\begin{array}{c}\text { Real } \\
0,5 \\
\end{array}$} & \multirow{2}{*}{$\begin{array}{c}\text { Realista } \\
0,3 \\
\end{array}$} & \multirow{2}{*}{$\begin{array}{c}\text { Pesimista } \\
0,1\end{array}$} & \multirow{2}{*}{$\begin{array}{c}\text { Ineficiencia } \\
0\end{array}$} \\
\hline 34 & Impacto social & & & & & \\
\hline 35 & $\begin{array}{l}\text { Incremento de productividad por in- } \\
\text { vestigación gran nacional }\end{array}$ & 1 & 0,4 & 0,1 & 0,05 & 0 \\
\hline 36 & $\begin{array}{l}\text { Incremento de productividad por in- } \\
\text { vestigación gran trasnacional }\end{array}$ & 1 & 0,5 & 0,2 & 0,05 & 0 \\
\hline 37 & $\begin{array}{l}\text { Incremento de productividad por in- } \\
\text { vestigación mediana }\end{array}$ & 1 & 0,8 & 0,4 & 0,1 & 0 \\
\hline 38 & $\begin{array}{l}\text { Incremento de productividad por in- } \\
\text { vestigación micro y pequeña }\end{array}$ & 1 & 0,9 & 0,5 & 0,2 & 0 \\
\hline 39 & Infraestructura CI & 1 & 0,8 & 0,4 & 0,2 & 0 \\
\hline 40 & Infraestructira IES & 1 & 0,7 & 0,3 & 0,1 & 0 \\
\hline 41 & Innovación & 1 & 0,5 & 0,2 & 0,05 & 0 \\
\hline 42 & Inserción laboral & 1 & 0,6 & 0,3 & 0,1 & 0 \\
\hline 43 & Inserción laboral en CI & 1 & 0,9 & 0,7 & 0,4 & 0 \\
\hline 44 & Inserción laboral en IES & 1 & 0,9 & 0,7 & 0,4 & 0 \\
\hline 45 & Inserción nivel posgrado & 1 & 0,6 & 0,3 & 0,1 & 0 \\
\hline 46 & Investigadores en el SNI & 1 & 0,9 & 0,6 & 0,2 & 0 \\
\hline 47 & Investigadores no SNI & 1 & 0,8 & 0,6 & 0,2 & 0 \\
\hline 48 & Maestría & 1 & 0,7 & 0,3 & 0,1 & 0 \\
\hline 49 & Negocios & 1 & 0,5 & 0,2 & 0,05 & 0 \\
\hline 50 & Otras CI & 1 & 0,8 & 0,4 & 0,2 & 0 \\
\hline 51 & Otras IES & 1 & 0,8 & 0,4 & 0,2 & 0 \\
\hline 52 & Reconocimiento internacional & 1 & 0,8 & 0,4 & 0,1 & 0 \\
\hline 53 & Reconocimiento nacional & 1 & 0,8 & 0,4 & 0,1 & 0 \\
\hline 54 & Reinversión en CI & 1 & 0,5 & 0,2 & 0,05 & 0 \\
\hline 55 & Reinversión en IES & 1 & 0,3 & 0,1 & 0,05 & 0 \\
\hline 56 & Reinversión privada & 1 & 0,6 & 0,3 & 0,1 & 0 \\
\hline 57 & Reinversión pública & 1 & 0,6 & 0,3 & 0,1 & 0 \\
\hline 58 & Utilidad social & 1 & 0,7 & 0,3 & 0,1 & 0 \\
\hline 59 & Valor agregado personal & 1 & 0,8 & 0,4 & 0,1 & 0 \\
\hline 60 & Valoración empresarial & 1 & 0,8 & 0,4 & 0,1 & 0 \\
\hline
\end{tabular}

Fuente: elaboración propia.

Nota: Los valores presentados en esta tabla son resultado de la opinión de un conjunto de líderes de opinión trabajando en la región de Querétaro en los sectores académico, industrial, gubernamental y financiero. Se recabaron opiniones de líderes de cada uno de los sectores y se obtuvo el valor promedio para cada uno de los factores y escenarios considerados.

Es importante hacer notar que los valores de esta tabla permiten analizar escenarios de la influencia que la investigación científica y la educación superior tienen en el sistema de innovación de la región y que pueden ser modificados, precisamente, para simular escenarios que permitan tomar decisiones sobre cuánto y dónde invertir para el desarrollo científico y tecnológico. 
En la tabla III se presentan los resultados obtenidos en los seis escenarios mencionados anteriormente, considerando como entrada una unidad monetaria de financiamiento para ciencia y tecnología expresada en unidad monetaria por año (\$/año). Para los diferentes escenarios, se presentan los resultados para la variable intermedia, recursos para proyectos de investigación y las variables de salida, generación de recursos académicos, impacto económico e impacto social.

TABLA III

\section{Comparación de escenarios para el SIRQ}

\begin{tabular}{|c|c|c|c|c|}
\hline Escenarios & $\begin{array}{c}\text { Recursos } \\
\text { para proyectos } \\
\text { de investigación } \\
(\$ / \text { año })\end{array}$ & $\begin{array}{l}\text { Generación } \\
\text { de recursos } \\
\text { académicos } \\
\text { (\$/año) }\end{array}$ & $\begin{array}{c}\text { Impacto } \\
\text { económico } \\
(\$ / a \tilde{n} o)\end{array}$ & $\begin{array}{c}\text { Impacto } \\
\text { social } \\
(\$ / a n ̃ o)\end{array}$ \\
\hline OPTIMISTA A & 1,000 & 1,500 & 61,00 & 63,50 \\
\hline OPTIMISTA B & 1,000 & 1,500 & 30,00 & 32,50 \\
\hline REAL & 0,918 & 0,369 & 9,753 & 5,214 \\
\hline REALISTA & 0,312 & 0,008 & 1,383 & 0,417 \\
\hline PESIMISTA & 0,100 & $1,25 \times 10-5$ & 0,066 & 0,007 \\
\hline INEFICIENCIA & 1,000 & 0 & 0 & 0 \\
\hline
\end{tabular}

Fuente: elaboración propia.

Al realizar la comparación de los seis escenarios propuestos se observa lo siguiente para el SIRQ:

a) Por mucho el escenario optimista A resulta ser el óptimo, pues por cada 1 \$/año invertido en investigación científica y tecnológica, se tiene como beneficio económico 61 \$/año y como beneficio social 63,5 \$/año.

b) La única diferencia entre el escenario optimista A y B, es que en el A se tiene una interconectividad interinstitucional que trabaja con eficiencia. Tal y como se observa, la existencia de instituciones organizadas con objetivos y metas comunes, trae como consecuencia una duplicación de los beneficios obtenidos, tal y como se asume en el escenario A. Sin interconectividad institucional, el escenario B, sólo obtiene 30 \$/año de impacto económico y 32,5 \$/año de impacto social.

c) Ambos escenarios optimista A y B generan recursos académicos por 1,5 \$/año por cada 1 \$/año invertido en ciencia y tecnología, el resultado es el mismo para ambos casos. Aunque podría esperarse que el resultado fuera el doble para el escenario A, esto no sucede, debido a que la interconectividad es muy importante para conseguir impacto, ya sea social o económico, no así para el sector académico, que tiene su propia dinámica y puede sobrevivir como un "lujo académico». 
d) Tanto el escenario optimista A como el B, son escenarios ideales a los que difícilmente se tendrá acceso de forma inmediata.

e) De acuerdo a las consideraciones realizadas, el objetivo a fijarse es el modelo real, el cual podría llegar a alcanzarse bajo ciertas situaciones y compromisos por parte de los actores del SIRQ.

f) En el escenario real, aunque se invierte 1 \$/año, al entrar en el SIRQ se convierte en 0,918 \$/año, que produce 0,369 \$/año en generación de recursos académicos, 9,753 \$/año en impacto económico y 5,214 \$/año en impacto social. Lo cual comparado con los escenarios A y B es pobre, sin embargo, es plausible y factible, observándose buenos beneficios para la región.

g) Se puede considerar que el escenario realista es el que vive la región de Querétaro actualmente, donde por cada 1 \$/año invertido, se tiene 0,008 \$/año en generación de recursos académicos, 1,383 \$/año en impacto económico y 0,417 \$/año en impacto social. Lo cual se ve reflejado en la región: academia activa, pero económicamente improductiva, economía industrial activa desvinculada de la academia y escaso impacto social. Lo anterior refleja el concepto de que bajo las condiciones actuales de funcionamiento del SIRQ, la investigación a nivel posgrado es un «lujo académico».

b) Los escenarios pesimista y de ineficiencia total, ilustran lo catastrófico de la situación, de seguir actuando aislados el gobierno, la academia, la industria y el sector financiero.

Los resultados obtenidos del modelo desarrollado, coinciden fielmente con la situación de la investigación en el SIRQ, considerando lo que reflejan los indicadores académicos sociales y económicos de la región (CONACYT, 2007), por lo que se estima posible su aplicación a nivel nacional.

\section{Bibliografía}

Alfonso, O.; Monteiro, S.; Thompson, M. (2010): A Growth model for the Quadruple Helix Innovation Theory. Working Paper Series. NIPE WP, 12/2010, Universidad de Mioho.

Braczyk, H. J.; Cooke, P.; Heidenreich, M. (ed.). (1998): Regional innovation systems: the role of governance in a globalized world. London and Pennsylvania: UCL Press.

Breschi, S.; Malerba, F. (1997): Sectoral innovation systems: technological regimes, Schumpeterian dynamics, and spatial boundaries. En: Edquist, C. (ed.) Systems of Innovation: Technologies, Institutions and Organizations. London and Washington: Pinter/Cassell Academic.

Carlsson, B. (ed.) (1995): Technological systems and economic performance: the case of factory automation. Dordrecht: Kluwer.

CONACYT. (2007): Estado del Arte de los Sistemas Estatales de Ciencia y Tecnología. Querétaro. Dirección Adjunta de Desarrollo Regional y Sectorial. Dirección de Desarrollo Estatal. 
Cooke, P.; Gómez Uranga, M.; Etxebarría, G. (1997): Regional systems of Innovation: Institutional and Organizational Dimensions, Research Policy, 26, 475-491.

Desai, N. (2006): A Framework for Technology Transfer. PICMET Proceedings, 1781-1788.

Edquist, C. (ed.) (1997): Systems of Innovation: Technologies, Institutions and Organizations. London: Pinter Publisher, Cassell Academics.

Edquist, C. (2001): Systems of Innovation for Development (SID). Background paper for the UNIDO World Industrial Development Report (WIDR), written for Investment Promotion and Institutional Capacity-building division, Industrial Policies and Research Branch, United Nations Industrial Development Organization (UNIDO), January 2001.

Edquist, C.; Mckelvey, M. (ed.) (2000): Systems of Innovation: Growth, Competitiveness and Employment. An Elgar Reference Collection (two volumes), Cheltenham: Edward Elgar.

Etzkowitz, H.; Leydesdorff, L. (2000): The dynamics of innovation: from National System and Mode 2 to a Triple Helix of University - industry - government relations. Research Policy, 29, 109-123.

Freeman, C. (1988): Japan: A new national innovation system. En: Dosi, G.; Freeman, C.; Nelson, R.; Silverberg, G.; Soete, L. (eds.) Technology and Economy Theory. London: Pinter Publishers, 330-348.

Freeman, C. (1995): The National System of Innovation in Historical Perspective. Cambridge Journal of Economics, vol. 19 (1), 5-24.

Lozano, A. (2003): Industria, Vinculación, Academia. NTHE, Consejo de Ciencia y Tecnología del Estado de Querétaro, boletín núm. 17, 5-10.

Lozano, A. (2008): Desarrollo de la Ciencia y la Tecnología en Querétaro, Horizonte a 25 años. NTHE, Consejo de Ciencia y Tecnología del Estado de Querétaro, boletín núm. 25, 3-15.

Lozano, A. (2009): Vinculación, Algunos Casos de Éxito. NTHE, Consejo de Ciencia y Tecnología del Estado de Querétaro, boletín 26, 3-14.

Lundvall, B. A. (1985): Product innovation and user-producer interaction, industrial development. Research Series 31, Aalborg: Aalborg University Press.

Lundvall, B. A.; Nielsen, P. (2007): Knowledge management and innovation performance. International Journal of Manpower, 28 (4), 207-223.

Muñoz, A.; Lozano, A.; Bernal, M. C. (2001): Vinculación, algunos aspectos que ilustran la problemática para establecer la relación Academia-Industria. Consejo de Ciencia y Tecnología del Estado de Querétaro, p. 5-22.

Nelson, R. (1993): National Innovation Systems: A comparative analysis. Oxford University Press, p. 541.

OCDE y EUROSTAT (2005): Manual de Oslo (3. ${ }^{a}$ ed.). París.

Rohrbeck, R.; Döhler M.; Arnold, H. M. (2009): Creating growth with externalization of R\&D results- he spin-along approach. Global Business and Organizational Excellence, 28, 44-51.

Sancho, R. (2007): Innovación Industrial. Revista Española de Documentación Científica, vol. 30 (4), 553-564. 